\title{
Graceful Planes and Thin Tunnel-Free Meshes
}

\author{
Valentin E. Brimkov and Reneta P. Barneva \\ Eastern Mediterranean University, \\ Famagusta, TRNC, Via Mersin 10, Turkey \\ \{brimkov, barneva\}.as@mozart.emu.edu.tr
}

\begin{abstract}
In this paper we present an approach to describe polyhedra by meshes of discrete triangles. The study is based on the theory of arithmetic geometry [10]. We introduce a new class of discrete planes (respectively lines) which we call graceful planes (respectively graceful lines). We use naive planes and graceful lines to obtain as thin as possible triangular mesh discretization admitting an analytical description. The interiors of the triangles are portions of naive planes, while the sides are graceful lines.
\end{abstract}

Key words: Discrete 3D modeling, Discrete planes, Discrete lines, Discrete triangles, Mesh of triangles.

\section{Introduction}

A promising approach in discrete geometry for computer imagery is the one based on arithmetic geometry $\mathbf{1}^{1}$. Reveillès observed in his pioneer work [10] that discrete straight lines in the plane can be analytically defined through a double linear Diophantine inequality of the form $0 \leq a x+b y+\mu<\omega$, where the parameters are all integers. The parameter $\omega>0$ is interpreted as a thickness of the discrete line, while $\mu$ is the internal translation constant which measures the shift of the line with respect to the origin. Subsequently this definition has been naturally extended to define discrete planes $0 \leq a x+b y+c z+\mu<\omega$, where the parameters $\mu$ and $\omega$ have the same interpretation. In recent years, arithmetic geometry has been developed by many authors (see for example the bibliography in [9]). The main objective is to obtain simple analytical description of the basic Euclidean primitives (lines, segments, triangles, circles, planes, spheres, etc.) and, on this basis, to create tools for efficient modeling of more sophisticated objects composed by such primitives. (See 221 for related discussion.) The approach of arithmetic geometry is very successful when dealing with linear objects, such as lines, planes, or their portions. This is of importance from a practical point of view since for various applications, it is sufficient to obtain good polyhedral approximation to a given real object. Thus it is an important task to study the properties of the above mentioned linear primitives, and to develop new methods for modeling through such primitives.

${ }^{1}$ Sometimes the terms "analytic discrete geometry" or "digital geometry" are used instead. 
In 3D modeling the discrete planes play a central role. The discretized objects are usually represented as meshes of polygonal (usually triangular) patches of discrete planes. A principal requirement for the obtained discretization is to be tunnel-free, i.e., to have no hole of certain type. The best studied classes of discrete planes are the naive planes (with thickness $\omega=\max \{|a|,|b|,|c|\}$ ), and the standard planes (with thickness $\omega=|a|+|b|+|c|$ ). These two classes possess interesting and useful properties. For instance, the naive planes are the thinnest possible tunnel-free discrete planes [1. However, naive planes have almost never been used for modeling purposes, due to certain theoretical obstacles which are difficult to overcome. For example, the intersection of two naive planes may be a set which is too far from the intuitive idea of line and, in fact, it may be a disconnected set of voxels (see corresponding examples in [75]).

In a recent work [5] we have studied the possibilities of using naive planes for triangular mesh discretization. We have proposed a general discretization scheme and, within its framework, we have shown how to obtain a thin tunnelfree approximation to a mesh of two triangles with a common side. This solution might be quite satisfactory regarding any practical purposes. However, in one particular degenerate case the solution is necessarily algorithmic rather than analytical. In the present paper we continue and extend our investigation with the aim to obtain a way for truly analytical discrete modeling, through the thinnest possible discrete planes.

We approach the problems of generating 3D polygons (triangles, for definiteness), segments of 3D discrete lines, as well as meshes of 3D discrete triangles. For this purpose, we define a new class of discrete planes which we call graceful planes (Section 3). The latter can be classified between the naive and the standard planes, and have the important property of being the thinnest possible discrete planes such that any (appropriately defined) "linear segment" of any graceful plane is a connected set of voxels. On the basis of graceful planes we define $3 \mathrm{D}$ discrete lines, called graceful lines, which are portions of graceful planes. One can use graceful lines to approximate the sides of 3D triangle, while the rest (interior) of the triangle can be modeled through naive planes (Section 4). Thus we obtain a thin tunnel-free triangular mesh discretization with analytical description (Section 5). We conclude with some remarks in Section 6 .

\section{Preliminaries}

In this section we recall some definitions and facts from discrete geometry, which will be used in the sequel. For a detailed account of the matter, the reader is referred to [10,5].

\subsection{Definitions and Facts}

Throughout $\lfloor x\rfloor$ denotes the greatest integer not exceeding the real number $x$, while $\left\{\frac{p}{q}\right\}=p-q\left\lfloor\frac{p}{q}\right\rfloor$ is the Euclidean remainder of the rational number $\frac{p}{q}$. 
Discrete coordinate plane/space consists of unit squares/cubes, called pixels/voxels, centered on the integer points of the Cartesian coordinate system in the plane/space, and with edges parallel to the coordinate axes. In the discrete coordinate plane two pixels are 4-adjacent if they share a common side, and they are 8-adjacent if they have a common side or vertex. A discrete line $L(a, b, \mu, \omega): 0 \leq a x+b y+\mu<\omega$ is naive if $\omega=\max \{|a|,|b|\}$, standard if $\omega=|a|+|b|$, and ${ }^{*}$-connected if $\max \{|a|,|b|\}<\omega<|a|+|b|[10]$. If $a$ and $b$ are both nonzero, then any two neighboring pixels of a standard line are 4connected, while a *-connected line has always two pixels which are 8-adjacent but not 4-adjacent.

Any 2D naive line $L$ is functional on one of the coordinate axes, i.e., for every fixed $x$ (every fixed $y) \in \mathbf{Z}$ there exists exactly one pixel belonging to $L$. A special case of naive line providing the best discrete approximation to the corresponding continuous straight line among all the possible naive lines is the Bresenham line 4. This line admits an analytical description. Given an Euclidean line $a x+b y+c=0$, the relevant Bresenham line can be defined as a naive line $L\left(a, b, c+\left\lfloor\frac{\omega}{2}\right\rfloor, \omega\right)$, where $\omega=\max \{|a|,|b|\}$.

Two discrete lines are called equivalent if they are equal within translation.

In the $3 \mathrm{D}$ space, two voxels are 6 -adjacent if they share a common face. The voxels are 18-adjacent if they have a common edge or face. They are 26-adjacent if they have a common vertex, edge, or face. A sequence of voxels is a $k$-path $(k=6,18,26)$ if every two consecutive voxels along the sequence are $k$-adjacent. Two voxels are $k$-connected $(k=6,18,26)$ if there exists a $k$-path between them. A set of voxels is connected if there exists at least a 26-path between every two voxels. Otherwise it is disconnected.

The plane $P(a, b, c, \mu, \omega): 0 \leq a x+b y+c z+\mu<\omega$ has $k$-tunnel $(k=6,18,26)$ if there exist two $k$-adjacent voxels $A\left(x_{A}, y_{A}, z_{A}\right)$ and $B\left(x_{B}, y_{B}, z_{B}\right)$ such that $a x_{A}+b y_{A}+c z_{A}+\mu<0$ and $a x_{B}+b y_{B}+c z_{B}+\mu \geq \omega$. A discrete plane without any $k$-tunnels is said to be $k$-tunnel-free. For our purposes, we also need a definition of 6 -tunnels and 6-tunnel freedom in finite sets of voxels. Such a definition can be given in terms of algebraic topology. Specifically, one can consider a voxel as a closed unit cube centered at a point in the 3D space with integer coordinates. Given a finite set of voxels $S$, let $\cup S$ denote the polyhedron obtained as a union of the voxels in $S$. We say that $S$ has 6 -tunnels if $\cup S$ is not a simply connected set. Otherwise $S$ is 6 -tunnel free [5]. Usually a 6 -tunnel-free set is called tunnelfree for short. In a similar (although somewhat more involved) way one can also define 18- and 26-tunnels.

A discrete plane $P=P(a, b, c, \mu, \omega)$ is functional on a coordinate plane, say, $O x y$, if for any pixel $(x, y)$ from $O x y$ there is exactly one voxel belonging to $P$. The plane $O x y$ is called functional plane for $P(a, b, c, \mu, \omega)$ and denoted by $\pi_{P}$. It is a well known fact that a naive plane is functional on at least one of the coordinate planes $O x y, O y z$, or $O z x$ [7]. Moreover, if $P(a, b, c, \mu, \omega)$ is a naive plane with $|c|=\max (|a|,|b|,|c|)$ then $\pi_{P}=O x y$. 
Finally, we recall that a naive $3 D$ digital line [8] is defined as intersection of two discrete planes which is 26-connected set of voxels and such that removing any voxel separates the line into two 26 -connected components.

\section{2 "Naive" Triangles}

We first define a triangle in the $2 \mathrm{D}$ plane. Given an Euclidean triangle $\triangle A B C$ in $O x y$, the corresponding $2 \mathrm{D}$ discrete triangle $T=T_{2 D}(A, B, C)$ is determined as follows. $T$ consists of a border which is the set of pixels from the Bresenham line segments between the vertices $A, B$, and $C$ of $T_{2 D}(A, B, C)$, and an interior which is the set of all pixels whose centers belong to the interior of $\triangle A B C$ but do not belong to the border.

Three-dimensional ("naive") discrete triangle $T(A, B, C)$ is defined as a portion of a special kind of naive plane, called regular plane and denoted $P_{R}^{A B C}$, passing through the centers of three given voxels $A, B$, and $C$-vertices of the triangle. The projection of $T(A, B, C)$ over $\pi_{P_{R}^{A B C}}$ is a $2 \mathrm{D}$ discrete triangle $T_{2 D}\left(A^{\prime}, B^{\prime}, C^{\prime}\right)$, where $A^{\prime}, B^{\prime}$, and $C^{\prime}$ are the respective projections of $A, B$,

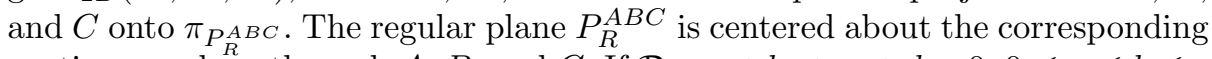
continuous plane through $A, B$, and $C$. If $\mathcal{P}: a x+b y+c z+d=0,0 \leq a \leq b \leq c$ is a continuous plane in the $3 \mathrm{D}$ space, then the corresponding regular plane is the naive plane $P_{R}=P\left(a, b, c, d+\left\lfloor\frac{\omega}{2}\right\rfloor, \omega\right)$.

A side of $T(A, B, C)$, say, $\overline{A B}$, is the set of voxels whose projections on $\pi_{P_{R}^{A B C}}$ constitute the segment of Bresenham line defined by $A^{\prime}$ and $B^{\prime} . \overline{A B}$ can be considered as a sort of discrete $3 \mathrm{D}$ line, which we call pseudoline [5].

\subsection{Generation Scheme}

The 3D discrete triangles defined above can be obtained through a simple generation scheme. Let $A, B$, and $C$ be a triple of points in the 3D space with integer coordinates, and let us consider them as vertices of a $3 \mathrm{D}$ discrete triangle. Our generating method adheres to the following scheme.

\section{Generation Scheme}

1. Determine the regular plane $P_{R}^{A B C}$ from which the discrete triangle is a portion.

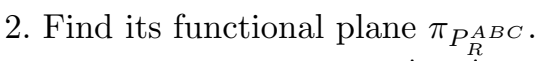

3. Find the projections $A^{\prime}, B^{\prime}$, and $C^{\prime}$ of the vertices $A, B$, and $C$ onto $\pi_{P_{R}^{A B C} \text {. }}$

4. Compute the $2 \mathrm{D}$ triangle with vertices $A^{\prime}, B^{\prime}$, and $C^{\prime}$ in $\pi_{P_{R}^{A B C}}$.

5. Generate the 3D triangle in $P$ from its projection over $\pi_{P_{R}^{A B C}}$.

Clearly, this scheme is readily adapted for generation of other 3D Euclidean primitives, such as lines, segments, arbitrary polygons, etc. It can also be easily modified for planes which are thicker than naive and thus not functional on any coordinate plane. Such a modification has been suggested in [5]. Specifically, a 
discrete plane $P$ with thickness $\omega$ greater than $c$ is considered as pseudofunctional on the coordinate plane $O x y$ corresponding to the largest coefficient $c$. Then the pseudofunctional plane can be used in the generation scheme instead of functional plane.

Let us mention that a similar way of generating the supercover of an Euclidean triangle (that is the set of all voxels intersected by the triangle) has been presented in [3].

\section{4 "Naive" Triangular Meshes}

Let us consider four voxels $A, B, C$, and $D$, such that $A, B$, and $C$ form one triangular patch, and $A, B$, and $D$ form another triangular patch. The pair of the edge-connected triangles $\triangle(A, B, C)$ and $\triangle(A, B, D)$ is called a mesh of two triangles.

Let $P_{R}^{A B C}$ and $P_{R}^{A B D}$ be the regular planes determined by the triples $A, B$, $C$ and $A, B, D$, respectively. Using the generation scheme from the preceding section, one can determine two discrete triangles $T(A, B, C)$ and $T(A, B, D)$, belonging to $P_{R}^{A B C}$ and $P_{R}^{A B D}$, respectively, and forming a mesh of discrete ("naive") triangles.

In has been noticed in [5] that the borders of a discrete triangle, as well as the discrete triangle itself, might be a disconnected set of voxels. If $P_{R}^{A B C}$ and $P_{R}^{A B D}$ are functional on the same coordinate plane, and both $T(A, B, C)$ and $T(A, B, D)$ are disconnected, the corresponding mesh may be disconnected, too. A disconnected discrete triangle (disconnected mesh of discrete triangles) admits a simple topological characterization provided by the following lemma.

Lemma 1. 5] Let the discrete triangle $T=T(A, B, C)$ be a portion of a discrete plane which is functional on Oxy. Then $T$ is disconnected if and only if there is at least one pair of voxels $v_{1}, v_{2} \in T$ such that the following conditions are met:

(a) There exists a vertex of $T$, say $A$, such that the projections of $v_{1}$ and $v_{2}$ onto Oxy belong to the Bresenham lines $\overline{A^{\prime} B^{\prime}}$ and $\overline{A^{\prime} C^{\prime}}$ in Oxy, determined by the corresponding projections $A^{\prime}, B^{\prime}$, and $C^{\prime}$ of $A, B$, and $C$.

(b) If $v_{1}=\left(x^{\prime}, y^{\prime}, z^{\prime}\right)$ and $v_{2}=\left(x^{\prime \prime}, y^{\prime \prime}, z^{\prime \prime}\right)$, then $\left|x^{\prime}-x^{\prime \prime}\right|=1,\left|y^{\prime}-y^{\prime \prime}\right|=1$, and $\left|z^{\prime}-z^{\prime \prime}\right|=2$;

(c) Without loss of generality, suppose that $v_{1}=\left(x^{\prime}, y^{\prime}, z^{\prime}\right), v_{2}=\left(x^{\prime}+1, y^{\prime}+\right.$ $\left.1, z^{\prime}+2\right)$. Then $T_{2 D}=T_{2 D}\left(A^{\prime}, B^{\prime}, C^{\prime}\right)$ does not contain the pixels $p^{\prime}=\left(x^{\prime}, y^{\prime}+1\right)$ and $p^{\prime \prime}=\left(x^{\prime}+1, y^{\prime}\right)$.

A pair of voxels $v_{1}, v_{2}$ satisfying the conditions of Lemma 1 is called point of disconnection of the discrete triangle $T$. A pair of voxels $v_{1}, v_{2}$ which belong to a pseudoline $g$ and satisfy condition (b) of Lemma 1 , is called point of disconnection of $g$. Clearly, a point of disconnection of $T$ is a point of disconnection of two of the sides of $T$ (which are pseudolines, by definition). 


\section{Graceful Planes and Lines}

In this section we introduce an important class of planes which will play a crucial role in our modeling methods. Throughout, for a given discrete plane $P(a, b, c, \mu, \omega)$, we will assume for the sake of simplicity that $0 \leq a \leq b \leq c$. We can also assume without loss of generality that $a, b$, and $c$ are relatively prime. We begin with a few remarks.

Assume first that $P$ has a thickness $\omega<c$, i.e., $P$ is "thinner" than a naive plane. Then $P$ may contain tunnels and, in general, may be a disconnected set of voxels. Therefore in constructing methods for discretization we will be interested in classes of planes of thickness $\omega \geq c$.

Let $P$ be of thickness $\omega=c$, i.e., a naive plane. Then $P$ is functional on Oxy. It is well known (see, e.g., 65]) that $P$ may contain a configuration of the form shown on Fig. 1. In this configuration there are two voxels $v$ and $v^{\prime}$, which correspond to pixels $p$ and $p^{\prime}$ having a common vertex, but the $z$-coordinates of $v$ and $v^{\prime}$ differ by 2 . We will call such a pair of voxels in a discrete plane a jump.

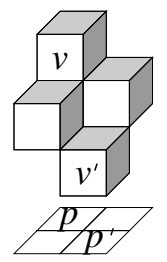

Fig. 1.Possible configuration of voxels in a naive plane.

Now let $P$ be of thickness $\omega>c$, i.e., "thicker" than a naive plane. It means that $P$ will not be functional on $O x y$, i.e., for certain pixels $(x, y)$ of $O x y, P$ will contain at least two voxels one on the top of the other. If for some pixel $(x, y)$ there are exactly two voxels $v=(x, y, z)$ and $v^{\prime}=(x, y, z+1)$ belonging to $P$, we will call the pair $v, v^{\prime}$ a tandem.

The following fundamental lemma will be our basic tool in developing discretization methods.

Lemma 2. A naive plane $P(a, b, c, \mu, \omega)$ has jumps if and only if $c<a+b$.

Proof. To prove the stated result, we will show that: If $c<a+b$ than the discrete plane $P$ has jumps; If $c \geq a+b$ then $P$ does not have any jumps.

Given a naive plane $P(a, b, c, \mu, \omega): 0 \leq a x+b y+c z+\mu<\omega$, consider a fixed value of $z: z=z_{0}$. For this value of $z$, the above double inequality becomes $0 \leq a x+b y+c z_{0}+\mu<\omega$, which is an equation of a discrete line $L_{z_{0}}$ with thickness $\omega$ and translation constant $\mu_{0}=\mu+c z_{0}$. It is clear that the naive plane $P$ has jumps if and only if for some $z_{0}$ the line $L_{z_{0}}$ is thinner than standard (see Fig. $2 \mathrm{a})$. 


\begin{tabular}{|l|l|l|l|l|l|}
\hline 5 & 5 & 6 & 6 & 7 & 7 \\
\hline 4 & 5 & 5 & 6 & 6 & 7 \\
\hline 4 & 4 & 5 & 5 & 6 & 6 \\
\hline 3 & 4 & 4 & 5 & 5 & 6 \\
\hline 2 & 3 & 3 & 4 & 4 & 5 \\
\hline 2 & 2 & 3 & 3 & 4 & 4 \\
\hline 1 & 2 & 2 & 3 & 3 & 4 \\
\hline 1 & 1 & 2 & 2 & 3 & 3 \\
\hline 0 & 0 & 1 & 1 & 2 & 2 \\
\hline
\end{tabular}

a)

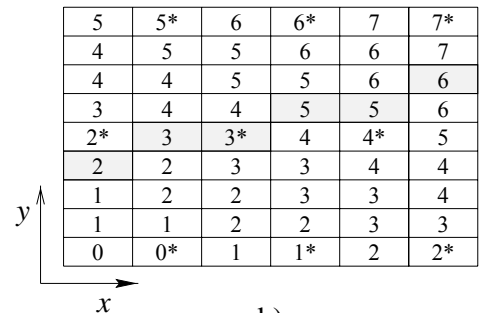

b)

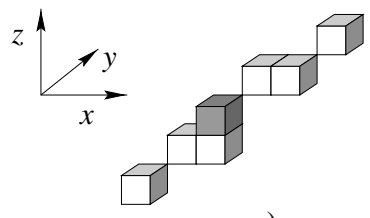

c)

Fig. 2. Illustration to the notions jump, graceful plane, and graceful line. In figures a) and b) every pixel is marked by the corresponding $z$-coordinate, as the lower-left corner corresponds to the voxel $(0,0,0)$. a) Naive plane $P(4,5,-8,4,8)$. For $z=4$ the corresponding discrete line in $O x y$ contains two 8-adjacent pixels, and at that point the plane has a jump between levels 3 and 5 . b) Graceful plane $P(4,5,-8,4,9)$. The pixels corresponding to tandems of voxels are marked by asterisk. Denotation $k *$ means that there are two voxels corresponding to that pixel, with $z$-coordinates $k$ and $k+1$, respectively. Apparently, these voxels form a lattice. c) Graceful line. It is a part of the given graceful plane $P(4,5,-8,4,9)$, and its projection is a naive line marked in Fig. $2 \mathrm{~b})$. Note that the pseudoline which could be built in the corresponding naive plane $P(4,5,-8,4,8)$ would be disconnected, as the voxel in dark gray would be missing.

Consider first the trivial case $\operatorname{gcd}(a, b)=1$. Under this condition, all the lines of the form $0 \leq a x+b y+c z_{0}+\mu<\omega$ are equivalent 2 . Therefore it suffices to consider an arbitrary such line. If $\omega=c<a+b$ then the line $0 \leq$ $a x+b y+c z_{0}+\mu<\omega$ is ${ }^{*}$-connected and hence it contains pixels $p_{1}$ and $p_{2}$ which are 8-adjacent but are not 4 -adjacent. Let $p_{1}^{\prime}$ and $p_{2}^{\prime}$ be the pixels for which both $p_{1}$ and $p_{2}$ are neighbors. Then the voxels from $P$ corresponding to $p_{1}^{\prime}$ and $p_{2}^{\prime}$ expose a jump.

If $\omega=c=a+b$ then the line $0 \leq a x+b y+c z_{0}+\mu_{0}<\omega$ is a standard line; if $\omega=c>a+b$ then it is a line thicker than standard. Clearly, in both cases the plane $P$ does not have any jumps.

Consider now the case when $\operatorname{gcd}(a, b)=d \neq 1$. Let us fix $a$ and $b$. Consider a naive plane $P(a, b, c, \mu, \omega)$ with $w=c<a+b$, i.e., $c=a+b-k$, where

${ }^{2}$ Theorem 3 [7] says that the intersection of a discrete plane $P(a, b, c, \mu, \omega)$ with the plane $z=z_{0}$ is a set of discrete lines; furthermore, for all integer values $z_{0}$ these discrete lines are equivalent. We notice that the second assertion of the theorem holds only if $\operatorname{gcd}(a, b)=1$, while for arbitrary $a, b$, and $c$ with $\operatorname{gcd}(a, b, c)=1$ such a statement is not valid. 
$1 \leq k \leq a$. Without loss of generality we consider only the extreme case $k=1$, i.e., $c=a+b-1$. In fact, all the planes $P(a, b, a+b-k, \mu, \omega), 1 \leq k \leq a$, can be considered as obtained from the plane $P(a, b, a+b-1, \mu, \omega)$ through cutting certain voxels. Obviously, this cannot lead to any loss of jumps.

The double inequality $0 \leq a x+b y+c z+\mu<\omega$ converts into $0 \leq a x+b y+(a+$ $b-1) z+\mu<a+b-1$. For any fixed $z$ this is a discrete line $L_{z}\left(a, b, \mu^{\prime}, \omega\right)$ with a translation constant $\mu^{\prime}=(a+b-1)+\mu$. We know from [3] that this discrete line is equivalent to a discrete line $L_{z}\left(a^{\prime}, b^{\prime}, \mu^{\prime \prime}, \omega^{\prime}\right)$, where $a^{\prime}=\frac{a}{d}, b^{\prime}=\frac{b}{d}, \mu^{\prime \prime}=\left\lfloor\frac{\mu^{\prime}}{d}\right\rfloor$, $\omega^{\prime}=\left\lfloor\frac{\omega}{d}\right\rfloor+1$ if $\left\{\frac{\omega}{d}\right\}>\left\{\frac{\mu^{\prime}}{d}\right\}$, and $\omega^{\prime}=\left\lfloor\frac{\omega}{d}\right\rfloor$ else. More in detail, $L_{z}$ is given by one of the following two double inequalities: $0 \leq \frac{a}{d} x+\frac{b}{d} y+\left\lfloor\frac{(a+b-1) z+\mu}{d}\right\rfloor<\left\lfloor\frac{a+b-1}{d}\right\rfloor$, or $0 \leq \frac{a}{d} x+\frac{b}{d} y+\left\lfloor\frac{(a+b-1) z+\mu}{d}\right\rfloor<\left\lfloor\frac{a+b-1}{d}\right\rfloor+1$.

In the latter case we have a standard line since its thickness is equal to $\left\lfloor\frac{a+b-1}{d}\right\rfloor+1=\left\lfloor\frac{a+b}{d}-\frac{1}{d}\right\rfloor+1=\frac{a+b}{d}-1+1=\frac{a}{d}+\frac{b}{d}=a^{\prime}+b^{\prime}$.

Regarding the former case, we will show that one can always find an integer $z$ for which

$$
\left\{\frac{(a+b-1) z+\mu}{d}\right\} \geq\left\{\frac{a+b-1}{d}\right\},
$$

and thus the first one of the above two cases holds. We have that $\left\{\frac{a+b-1}{d}\right\}=d-1$. Since any rational number modulo $d$ cannot exceed $d-1$, it follows that the only possibility is to have equality in (1).

Let us set $m=a+b-1$. We look for $z$ for which $m z+\mu=m(\bmod d)$, i.e., $m z=m-\mu(\bmod d)$. It is a well known fact from number theory that the above equation has a solution if and only if $\operatorname{gcd}(m, d)$ divides $m-\mu$. But it is so since $d=\operatorname{gcd}(a, b)$, hence $\operatorname{gcd}(m, d)$ divides $a$ and $b$. Then, in order to divide also $m=$ $a+b-1, \operatorname{gcd}(m, d)$ must be equal to 1 . Thus we obtained that the equation (1) has always an integral solution $z_{0}$. Since $\frac{a+b}{d}$ is integer, we have for the thickness of the corresponding discrete line $\left\lfloor\frac{a+b-1}{d}\right\rfloor=\left\lfloor\frac{a+b}{d}-\frac{1}{d}\right\rfloor \leq \frac{a+b}{d}-1<\frac{a}{d}+\frac{b}{d}$ $=a^{\prime}+b^{\prime}$, i.e., for $z=z_{0}$ the discrete line is ${ }^{*}$-connected and thus the discrete plane $P$ has jumps.

If $c \geq a+b$, i.e., $c=a+b+k$ for some integer $k \geq 0$, then clearly the corresponding discrete line $0 \leq a x+b y+(a+b+k) z+\mu \leq a+b+k$ for fixed $z$ will always be standard or thicker than standard, and therefore the plane $P$ will have no jumps.

Remark 3. Clearly if $\omega<c$ then the plane may have either tunnels or both jumps and tunnels. It is also easy to see that if $c<\omega<a+b$ then the plane has both jumps and tandems.

In the framework of our generation scheme, we are concerned with the study of possible disconnections of lines, triangles, and meshes. While Lemma 11 provides a structural description of the possible disconnections, the following lemmas provide a corresponding analytical characterization.

Lemma 4. Let $P(a, b, c, \mu, \omega)$ be a discrete plane and let $\overline{A B}$ be a discrete segment in $P$, obtained through the generation scheme applied to a naive discrete 
line $\overline{A^{\prime} B^{\prime}}$, where $A^{\prime}$ and $B^{\prime}$ are the projections onto $\pi_{P}$ of $A$ and $B$, respectively. Then $\overline{A B}$ can be disconnected if and only if $\omega<\max \{a+b, c\}$.

Proof. If $\omega<\max \{a+b, c\}$ then according to Lemma $2 P$ has jumps. Given a jump $v, v^{\prime}$ in $P$, let $p, p^{\prime}$ be the corresponding pixels in $\pi_{P}$. If now we choose any naive line in $\pi_{P}$ including the pixels $p$ and $p^{\prime}$, then obviously the corresponding discrete line segment in $P$ will be disconnected.

The "only if" part follows from the observation that a pseudoline in $P$ may be disconnected only if $P$ has jumps.

Lemma 5. Let a discrete plane $P(a, b, c, \mu, \omega)$ be given. A $3 D$ discrete triangle in $P$ can be disconnected if and only if $\omega<\max \{a+b, c\}$.

The proof is analogous to that one of Lemma 4. It is not difficult to see that Lemma 5 is still valid for polygons with arbitrary many vertices. The above discussion and the obtained properties lead us to introducing the following definition.

Definition 6. A discrete plane $P(a, b, c, \mu, \omega)$ with thickness $\omega=\max \{a+b, c\}$ is called a graceful plane.

The class of the graceful planes lies between the class of the naive and the standard planes. Note that if the maximum in the above definition is reached for $\omega=c$ then the graceful plane is a naive plane. Otherwise the graceful plane is a naive plane which in addition contains tandems. More precisely, for each value of $\omega$ between $c+1$ and $a+b$ a lattice of voxels is added. These lattices are equivalent and they belong to different parallel Euclidean planes, in correspondence to the concrete value of $w$. As a result, tandems appear in such a way that the obtained graceful plane is jump-free, and it is the thinnest possible discrete plane with this property. (See Fig. 2b).

Lemmas 4 and 5 show that the graceful planes are the thinnest possible discrete planes for which, in the framework of the generation scheme, Euclidean primitives like lines, segments and triangles (as well as arbitrary polygons) are always connected sets of voxels. This is an important property to be used further for modeling purposes.

Using the generation scheme, one can define a discrete line in a graceful plane. We will call such a line graceful line. (See Fig. 2c).

It is not difficult to see that any graceful line is either a naive $3 \mathrm{D}$ digital line or, otherwise, it may contain tandems. In contrast, the pseudolines defined earlier, which are obtained in the same way but on the basis of naive planes, may be connected or disconnected sets of voxels. In the former case they are naive $3 \mathrm{D}$ digital lines. In the latter case they can be considered as naive 3D digital lines with points of disconnection.

\section{Modeling of 3D Triangles}

As mentioned, the graceful planes can be used for modeling of Euclidean primitives. Based on the generation scheme, one can obtain connected and, thus, 
tunnel-free discretizations of segments and triangles. Regarding triangles or other polygons, the method can be refined in the following way.

\section{Graceful sides - Naive interior (GN-method)}

1. The sides of the triangle are approximated through the corresponding graceful lines.

2. The interior of the triangle is approximated through the corresponding portion of the relevant regular plane.

According to [5], a naive discrete triangle has tunnels only if it is disconnected. Keeping this in mind and using Lemma 5, it is easy to see that the discrete triangle obtained through the GN-method is connected and tunnel-free.

Another way for obtaining thin triangular approximation is the following.

\section{Standard-Naive sides - Naive interior (SNN-method)}

1. The projection of the sides onto the functional plane are standard lines, while the sides themselves are portions of naive planes.

2. The interior of the triangle is approximated through the corresponding portion of the relevant regular plane.

In view of Lemma 1, one can easily deduce that the obtained discrete polygon is connected and tunnel-free.

\section{$5 \quad$ Triangular Meshes Modeling}

We start this section with a fact similar to Lemmas 4 and 5 ,

Proposition 7. Let a mesh of two discrete triangles $T_{1}$ and $T_{2}$ be given, such that they are parts of discrete planes $P_{1}\left(a_{1}, b_{1}, c_{1}, \mu_{1}, \omega_{1}\right)$ and $P_{2}\left(a_{2}, b_{2}, c_{2}, \mu_{2}, \omega_{2}\right)$, respectively, with $\omega_{1} \geq c_{1}$ and $\omega_{2} \geq c_{2}$. Then the mesh can be disconnected if and only if $\omega_{1}<\max \left\{a_{1}+b_{1}, c_{1}\right\}$ and $\omega_{2}<\max \left\{a_{2}+b_{2}, c_{2}\right\}$.

The proof is similar to those of Lemmas 4 and 5 This proposition shows that the generation scheme applied directly to discrete planes thinner than graceful does not always produce a tunnel-free discretization. Below we present two approaches for obtaining thin discrete tunnel-free triangular meshes. The methods rely on appropriate modifications of the generation scheme and use the methods for triangle modeling given in Section 4. The following proposition provides an approach for tunnel-free triangular mesh modeling.

Proposition 8. Let a mesh of two discrete triangles $T_{1}$ and $T_{2}$ be constructed according to the generation scheme in such a way that they are portions of graceful planes. Then the mesh is connected and tunnel-free. 
The proof is analogous to one of Theorem 9 .

The method based on Proposition 8 can be refined on the basis of the GNmethod for triangle discretization. Accordingly, every triangle is approximated through the GN-method. We have the following theorem (which, in particular, implies Proposition 8).

Theorem 9. Discrete triangular mesh obtained applying the GN-method to all triangular patches, is tunnel-free.

Proof. Consider a mesh of two discrete triangles $T_{1}=T_{1}(A, B, C)$ and $T_{2}=$ $T_{2}(A, B, C)$ obtained through the GN-method, in the framework of the generation scheme. Assume first that $P_{R}^{A B C}$ and $P_{R}^{A B D}$ are functional on the same coordinate plane. In view of Lemma 5 each of the discrete triangles $T_{1}$ and $T_{2}$ is connected and tunnel-free. Moreover, both triangles have the same common side and therefore the mesh $T_{1} \cup T_{2}$ is tunnel-free, too.

Consider now the case where $P_{R}^{A B C}$ and $P_{R}^{A B D}$ are functional on different coordinate planes. Similar to the previous case, both discrete triangles are connected and tunnel-free. It has been shown in [5] (Theorem 5) that, under the latter condition, the mesh of the corresponding naive discrete triangles is connected and tunnel-free. Hence the mesh $T_{1} \cup T_{2}$ of triangles with naive interiors and graceful sides will be tunnel-free, as well.

Another approach of obtaining thin triangular mesh approximation can be based on the SNN-method. Accordingly, every triangular patch is approximated through the SNN-method. We have the following theorem.

Theorem 10. Discrete triangular mesh obtained applying the SNN-method to all triangular patches, is tunnel-free.

Proof. Let $T_{1}=T_{1}(A, B, C) \subset P_{R}^{A B C}$ and $T_{2}=T_{2}(A, B, D) \subset P_{R}^{A B D}$ be discrete triangles obtained through the SNN-method. In the case when $P_{R}^{A B C}$ and $P_{R}^{A B D}$ are functional on different coordinate planes the proof is identical to that one of Theorem 9

Consider the case when $P_{R}^{A B C}$ and $P_{R}^{A B D}$ are functional on the same coordinate plane. Since the projections of the triangles' sides onto the common coordinate plane are standard lines, then it is easy to see that each of the discrete triangles $T_{1}$ and $T_{2}$ fails the condition (c) of Lemma 1. Thus $T_{1}$ and $T_{2}$ are connected and tunnel-free, and they have a common side $\overline{A B}$. Consequently, the mesh $T_{1} \cup T_{2}$ will be tunnel-free.

It is clear that a discrete side can be analytically described through two double linear inequalities of the form $0 \leq a x+b y+c z+\mu<\omega$ and $0 \leq$ $a^{\prime} x+b^{\prime} y+\mu^{\prime}<\omega^{\prime}$.

It is also easy to see that the presented generation methods can be organized in such a way that their time complexity will be linear with respect to the number of voxels generated, which can be represented in a 2D array. (See [35] for a detailed discussion on a similar matter.) 


\section{Concluding Remarks}

In this paper we have defined a new class of discrete planes "sandwiched" between the naive and standard planes. On the basis of these planes, one can construct thin discrete triangular tunnel-free meshes admitting analytical description.

It would be interesting to look for other reasonable discretization methods providing optimally thin tunnel-free approximation to a polyhedral surface. It would be also a challenging task to develop relevant methods for obtaining thin discretizations of various $2 \mathrm{D}$ curves (e.g., the conic sections) and $3 \mathrm{D}$ surfaces (e.g., the quadratic surfaces).

\section{Acknowledgments}

We thank Jean Françon and Philippe Nehlig for several interesting and helpful discussions. We are indebted to the referees for their useful remarks and suggestions.

\section{References}

1. Andres, E., Acharya, R., Sibata, C.: Discrete Analytical Hyperplanes. GMIP 59 (1997) 302-309

2. Andres, E., Jacob, M.-A.: The Discrete Analytical Hyperspheres. IEEE TVCG 3 (1997) $75-86$

3. Andres, E., Nehlig, Ph., Françon, J.: Supercover of Straight Lines, Planes, and Triangles. In: Ahronovitz, E., Fiorio, Ch. (eds.): Discrete Geometry for Computer Imagery. Springer-Verlag, Berlin Heidelberg New York (1996) 243-254

4. Bresenham, J.E.: Algorithm for Computer Control of a Digital Plotter. ACM Trans. on Graphics 4 (1965) 25-30

5. Barneva, R.P., Brimkov, V.E., Nehlig, Ph.: Thin Discrete Triangular Meshes. Tech. Rep. RR 98/01, LSIIT, Strasbourg (1998). To appear in Theor. Comp. Sc. (Elsevier)

6. Debled-Renesson, I.: Reconnaissance des Droites et Plans Discrets. Thèse de doctorat, Université Louis Pasteur, Strasbourg (1995)

7. Debled-Renesson, I., Reveillès, J.-P.: A New Approach to Digital Planes. In: Spie's International Symposium on Photonics for Industrial Applications, Technical Conference Vision Geometry 3, Boston (1994)

8. Figueiredo, O., Reveillès, J.-P.: A Contribution to 3D Digital Lines. In: Discrete Geometry for Computer Imagery, 5th Int. Workshop, Clermont-Ferrand (1995) 187198

9. Françon J.: On Recent Trends in Discrete Geometry in Computer Imagery. In: Miguet, S., Montanvert, A., Ubeda, S. (eds.): Discrete Geometry for Computer Imagery. Lecture Notes in Computer Sciences, Vol. 1176. Springer-Verlag, Berlin Heidelberg New York (1996) 141-150

10. Reveillès, J.-P.: Géométrie Discrète, Calcul en Nombres Entiers et Algorithmique. Thèse d'état, Université Louis Pasteur, Strasbourg (1991) 